\title{
Anthocyanins increase serum adiponectin in newly diagnosed diabetes but not in prediabetes: a randomized controlled trial
}

\author{
Liping Yang ${ }^{1,2^{*}} \mathbb{0}$, Wenhua Ling ${ }^{2}$, Yun Qiu², Yong Liu ${ }^{1}$, Li Wang ${ }^{1}$, Jing Yang ${ }^{1}$, Changyi Wang ${ }^{1}$ and Jianping Ma ${ }^{1}$
}

\begin{abstract}
Background: Epidemiological studies have suggested that adiponectin is associated with the development of insulin resistance and type 2 diabetes. This study first examined the effect of purified anthocyanins, a group of dietary flavonoids, on serum adiponectin in patients with prediabetes and newly diagnosed diabetes.

Methods: A total of 160 patients with prediabetes $(n=90)$ or newly diagnosed diabetes $(n=70)$ were randomly assigned to either the anthocyanins group or the placebo group for 12 weeks of intervention.Serum adiponectin, a set of biomarkers related to glucolipid metabolism, anthropometric parameters, dietary intake and physical activity were measured before and after intervention.

Results: Anthocyanins increased serum adiponectin compared with placebo (net change $0.46 \mu \mathrm{g} / \mathrm{mL}, 95 \% \mathrm{Cl}[0.03$, $0.90], p=0.038$ ) in the subjects with newly diagnosed diabetes. No significant difference in the change in adiponectin was observed between the two groups either in the overall subjects $(0.02 \mu \mathrm{g} / \mathrm{mL}[-0.32,0.36], p=0.906)$ or in prediabetes $(-0.35 \mu \mathrm{g} / \mathrm{mL}[-0.85,0.16], p=0.174)$. Anthocyanins also decreased fasting glucose $(-0.5 \mathrm{mmol} / \mathrm{L}[-1$, $-0.04], p=0.035)$ in the subjects with newly diagnosed diabetes, but no such change was observed in those with prediabetes.

Conclusions: Anthocyanins supplementation for 12 weeks improved serum adiponectin and fasting glucose in patients with newly diagnosed diabetes, but not in patients with prediabetes.

Trial registration: ClinicalTrials.gov, NCT02689765. Registered on 6 February 2016, https://clinicaltrials.gov/ct2/show/ NCT02689765.
\end{abstract}

Keywords: Anthocyanin, Adiponectin, Prediabetes

\section{Highlights}

The First study to examine the effects of purified anthocyanins on serum adiponectin in patients with prediabetes and newly diagnosed diabetes.
Anthocyanins increased serum adiponectin and decreased fasting glucose in patients with newly diagnosed diabetes, but not in prediabetes.

Provide new evidence for anthocyanins in the treatment and prevention of type 2 diabetes.

*Correspondence: yanglp6@mail2.sysu.edu.cn

${ }^{1}$ Center for Chronic Disease Control, NanShan, Shenzhen, People's Republic of China

Full list of author information is available at the end of the article

\section{Introduction}

The prevalence of type 2 diabetes mellitus (T2DM) in conjunction with its cardiovascular complications is becoming the most serious health challenge worldwide. Adiponectin, the most common adipokine, has been

(c) The Author(s) 2020. This article is licensed under a Creative Commons Attribution 4.0 International License, which permits use, sharing, adaptation, distribution and reproduction in any medium or format, as long as you give appropriate credit to the original author(s) and the source, provide a link to the Creative Commons licence, and indicate if changes were made. The images or other third party material in this article are included in the article's Creative Commons licence, unless indicated otherwise in a credit line to the material. If material is not included in the article's Creative Commons licence and your intended use is not permitted by statutory regulation or exceeds the permitted use, you will need to obtain permission directly from the copyright holder. To view a copy of this licence, visit http://creativeco mmons.org/licenses/by/4.0/. The Creative Commons Public Domain Dedication waiver (http://creativecommons.org/publicdomain/ zero/1.0/) applies to the data made available in this article, unless otherwise stated in a credit line to the data. 
suggested to exert antiatherogenic and anti-inflammatory activities in various cardiometabolic diseases. It is also known to be an important regulator of insulin sensitivity and glucose metabolism. A previous meta-analysis conclusively showed a strong and consistent correlation between higher circulating adiponectin levels and lower risk of T2DM [1]. Another recent meta-analysis showed that hypoadiponectinemia was associated with the development of T2DM [2]. Adiponectin has been proposed to be a therapeutic target and prognostic marker for cardiometabolic diseases [3]. However, there are many conflicting results about the associations between circulating adiponectin and the prognosis of T2DM [4-7]. To date, the complex interplay between adiponectin and T2DM is not fully understood, and the role of circulating adiponectin in the development of diabetes remains unclear.

Anthocyanins are a large group of phytochemicals, concentrated from dark fruits and vegetables and are associated with health benefits. A cross-sectional study suggested that higher anthocyanin intake was associated with lower peripheral insulin resistance, reduced levels of inflammatory markers and improved adiponectin concentrations [8]. Numerous interventional studies have revealed that anthocyanins or anthocyanin-rich extracts improve lipid profiles in cardiometabolic diseases, but few studies have evaluated the effect of anthocyanins on circulating adiponectin $[9,10]$. Only one study examined the effect of anthocyanins on serum adiponectin in patients with well-controlled type 2 diabetes, and anthocyanins were found to increase adiponectin and decrease fasting glucose [11]. Whether these effects could be extended to patients with prediabetes or untreated newly diabetes is unclear. Prediabetes or newly diagnosed diabetes are identified to avoid the influence of medications and are more appropriate to prospectively evaluate the efficacy of anthocyanins intervention. Therefore, the aim of this study was to evaluate the effect of purified anthocyanins supplementation on serum adiponectin levels in patients with prediabetes or newly diagnosed diabetes.

\section{Methods \\ Subjects}

Participants aged 40-75 years were recruited from local community at outpatient clinic of a hospital in Guangzhou city, China. Potential participants were initially screened by their recent fasting blood glucose records, and volunteers were further screened by a face-to-face interview and were given a 3-h $75 \mathrm{~g}$ oral glucose tolerance test (OGTT) to confirm their eligibility. Patients with prediabetes or newly diagnosed diabetes were eligible for this study. According to the diagnostic criteria of the American Diabetes Association (ADA) [12], prediabetes is an intermediate state in which subjects meet one of the following criteria: impaired fasting glucose (IFG, 5.6-6.9 mmol/L), impaired glucose tolerance (IGT, 2-h glucose 7.8-11.0 mmol/L), or a glycated hemoglobin A1c (HbA1c) level of 5.7-6.4\%. Newly diagnosed diabetes was diagnosed in subjects who exceeded the upper limit of the above criteria (fasting glucose $>6.9 \mathrm{mmol} / \mathrm{L}, 2$-h glucose $>11.0 \mathrm{mmol} / \mathrm{L}$, or $\mathrm{HbA} 1 \mathrm{c}>6.4 \%$ ), and had not taken any diabetic medications before the screening.

Participants were excluded if they had pre-existing diabetes, had a history of hypoglycemic medical treatment, acute or chronic infectious diseases, untreated thyroid disease, serious liver or kidney dysfunction, the use of glucocorticoids, or suffering from traumatic injury or undergoing surgery within 6 months before enrollment; were lactating or pregnant women; or were individuals with polycystic ovarian syndrome. This trial was approved by the Ethics Committee of Sun Yat-Sen University, and written informed consent was obtained from each participant before enrollment.

\section{Study design}

This study was a 12-week randomized placebo-controlled trial that was registered at ClinicalTrials.gov as NCT02689765. The primary outcome was change in serum adiponectin. Sample size calculation were based on the previous study using purified anthocyanins as supplements [11], in which the change in serum adiponectin was $1.2 \mu \mathrm{g} / \mathrm{mL}$ (SD $1 \mu \mathrm{g} / \mathrm{mL}$ ). We calculated that 60 participants (prediabetes or newly diagnosed diabetes) per group, would be enough to detect a significant change in adiponectin $(1 \mu \mathrm{g} / \mathrm{mL}, \mathrm{SD} 2 \mu \mathrm{g} / \mathrm{mL})$ at $80 \%$ power and an alpha level of 0.05 . Considering a $20 \%$ dropout rate, we planned to recruit 80 participants per group and a total of 160 participants in this study.

Eligible participants were randomly assigned to either the anthocyanins group $(n=80)$ or the placebo group $(\mathrm{n}=80)$. The allocation sequences were determined by a computer-generated random-numbers table. The anthocyanins group consumed two anthocyanin capsules (Medox; Polyphenols AS, Norway) twice daily for a total of $320 \mathrm{mg}$ anthocyanins. The dose of anthocyanins was determined based on our previous trials that were performed in patients with T2DM [11] and dyslipidemia [13]. The control group consumed two identically packaged placebo capsules twice daily.

The participants were asked to maintain their habitual diet and physical activities, and to avoid consuming anthocyanin-rich foods, such as berries and grapes, during the whole study. Dietary analyses were conducted at baseline and 12 weeks of the study. Subjects were asked to provide detailed 3-day food records, and they were guided by a trained investigator. Scheduled capsules were dispensed to the participants, 
and participants were asked to return any remaining capsules to the clinic every 2 weeks. Compliance was assessed by using a short questionnaire and counting their remaining capsules. When the subjects consumed fewer than $80 \%$ of the dispensed capsules, they were excluded from the trial.

\section{Outcome measures}

At baseline and at the end of intervention, height and weight were measured by trained staff, and body mass index (BMI) was calculated as weight divided by height squared $\left(\mathrm{kg} / \mathrm{m}^{2}\right)$. Waist circumference, hip circumference and blood pressure were measured by the same staff. Fasting blood samples were collected after fasting overnight, and a 3-h OGTT was performed (blood samples were collected at $30,60,120$, and $180 \mathrm{~min}$ after a $75 \mathrm{~g}$ glucose challenge).

Serum samples were centrifuged $\left(15 \mathrm{~min}, 3000 \times g, 4{ }^{\circ} \mathrm{C}\right)$ within $30 \mathrm{~min}$ after blood collection and were stored at $-80{ }^{\circ} \mathrm{C}$. Fasting serum adiponectin was measured using an ELISA kit with a solid phase two-site enzyme immunoassay (Mercodia, Uppsala, Sweden). The average intraand inter-assay coefficients of variation for adiponectin were 3.1 and $5.4 \%$, respectively.

HbA1c was analyzed by high-pressure liquid chromatography (HPLC) (Bio-Rad Laboratories, USA). Insulin and C-peptide were assayed by electrochemical luminescence (Roche Diagnostics, Indianapolis, USA). Laboratory analyses of blood glucose, lipid profiles (total cholesterol, triglycerides, high-density lipoprotein cholesterol, low-density lipoprotein cholesterol, apolipoprotein A-1 and apolipoprotein B), C-reactive protein (CRP), and safety variables (liver enzymes) were performed by using an automatic biochemical analyzer (Mindray BS600, Shenzhen, China) following standard protocols.

\section{Statistical analysis}

SPSS 22.0 (IBM Inc., Chicago, IL, USA) was used for statistical analyses, and R 3.5.3 (R Foundation, Vienna, Austria) was used for graphing. $P<0.05$ was considered significant. The per-protocol data set without the imputation of missing data was used for data analysis. We used the unpaired Student's $t$-test (for continuous variables) and Chi-squared test (for categorical variables) to evaluate the baseline differences between the anthocyanins and the placebo groups. An independent Student's $t$-test was used to compare the differences between the two groups after intervention. We assessed the differences before and after the intervention within each group by using a paired $t$-test. Multiple comparisons were statistically corrected by using the false discovery rate.

\section{Results}

\section{General characteristics of the subjects}

A total of 160 eligible participants (80 in each intervention group) with prediabetes $(\mathrm{n}=90)$ or newly diagnosed diabetes $(n=70)$ were included in this trial. The baseline characteristics of all the randomized participants are presented in Additional file 1: Table S1. Participants in the anthocyanins and placebo groups were comparable in terms of age, gender, education, lifestyle factors, dietary intake and physical activity. Twenty participants $(12.5 \%)$ were lost from the study and two participants (one each group) were in poor compliance, resulting in 138 patients finally analyzed. More dropouts were observed in the placebo group $(n=17)$ than in the anthocyanins group $(n=3)$, with response rates of $79 \%$ and $96 \%$, respectively. The flow chart presented in Fig. 1 shows the allocation and the numbers of patients ultimately available for each group.

More than $90 \%$ of the participants in each group consumed at least $85 \%$ of the supplements provided, and no significant difference was observed in the proportion of poor compliance between the two groups. Participants reported a total of 10 adverse events to the intervention, and the proportion of adverse events was not significantly different between the two groups (Additional file 1: Table S2). Three adverse events were reported among participants in the placebo group, including abdominal pain $(n=1)$, diarrhea $(n=1)$ and skin rash $(n=1)$; seven adverse events were reported among participants in the anthocyanins group, including black stool $(n=5)$, insomnia $(n=1)$ and dizziness $(n=1)$. Within the subgroup of prediabetes and newly diagnosed diabetes, comparisons of baseline values indicated that the subjects in the two intervention groups had similar adiponectin levels and metabolic parameters (Table 1). No significant difference was observed between the anthocyanins and the placebo groups in terms of dietary intake and nutrients at either baseline or study end (Additional file 1: Table S3).

\section{Changes in serum adiponectin}

After 12 weeks of intervention, serum adiponectin was reduced in both the placebo group $(-0.31 \mu \mathrm{g} / \mathrm{mL}$ $[-0.55,-0.06], p=0.015)$ and the anthocyanins group $(-0.29 \mu \mathrm{g} / \mathrm{mL}[-0.53,-0.05], p=0.021)$. No significant difference in the net change in serum adiponectin was observed between the anthocyanins and the placebo group among all subjects $(0.02 \mu \mathrm{g} / \mathrm{mL}[-0.32,0.36]$, $p=0.906$ ) (Fig. 2a).

In the subgroup with prediabetes, serum adiponectin was reduced in the anthocyanins group $(-0.51 \mu \mathrm{g} /$ $\mathrm{mL}[-0.86,-0.16], p=0.006)$ but not in the placebo group $(-0.17 \mu \mathrm{g} / \mathrm{mL}[-0.53,0.2], p=0.365)$, and the 
Confirmation for eligibility

by 3 -hour OGTT $(\mathrm{n}=226)$

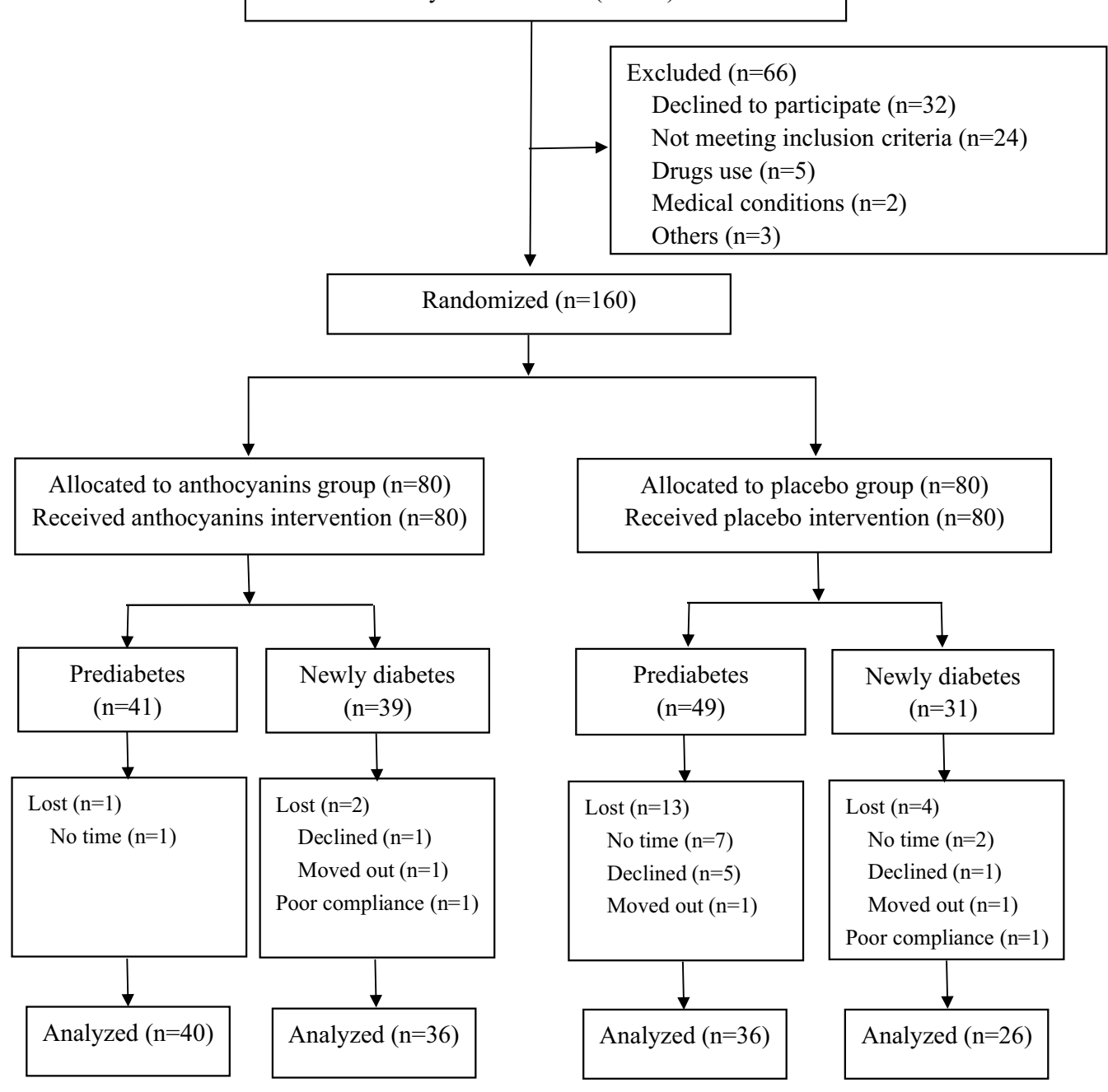

Fig. 1 The CONSORT flow diagram for participants

net change between the two groups was nonsignificant $(-0.35 \mu \mathrm{g} / \mathrm{mL}[-0.85,0.16], p=0.174)$ (Fig. 2b). In the subgroup with newly diagnosed diabetes, there was a significant reduction in serum adiponectin in the placebo group $(-0.48 \mu \mathrm{g} / \mathrm{mL}[-0.8,-0.16], p=0.004)$, whereas there was no change in the anthocyanins group $(-0.02 \mu \mathrm{g} / \mathrm{mL}[-0.33,0.3], p=0.917)$, and the net change in adiponectin between the two groups was statistically significant $(0.46 \mu \mathrm{g} / \mathrm{mL}[0.03,0.90], p=0.038)$ (Fig. 2c).

\section{Changes in markers for glucose and lipid metabolism}

Compared with placebo, anthocyanins decreased fasting glucose $(-0.5 \mathrm{mmol} / \mathrm{L}[-1,-0.04], p=0.035)$ in the subjects with newly diagnosed diabetes (Additional file 1 :
Table S4), increased serum apolipoprotein A-1 (apo A-1) $(0.15 \mathrm{~g} / \mathrm{L}[0.03,0.26], p=0.016)$ and decreased apolipoprotein B (apo B) $(-0.1 \mathrm{~g} / \mathrm{L}[-0.17,-0.03], p=0.008)$ in the subjects with prediabetes (Additional file 1: Table S5). Similar changes in apo A-1 and apo B were observed among all subjects. No significant change was observed in inflammatory markers (such as C-reactive protein), the anthropometric measurements (such as body weight, BMI, waist and hip circumferences), or blood pressure either among all subjects or among the subgroups (data available upon request). 
Table 1 Baseline values of adiponectin and glucolipid metabolic parameters in the anthocyanins and placebo group for the participants classified with prediabetes and newly diabetes

\begin{tabular}{|c|c|c|c|c|c|c|}
\hline & \multicolumn{3}{|l|}{ Prediabetes } & \multicolumn{3}{|l|}{ Newly diabetes } \\
\hline & Anthocyanins $(n=41)$ & Placebo $(n=49)$ & $P$ & Anthocyanins $(n=39)$ & Placebo $(n=31)$ & $P$ \\
\hline Adiponectin ( $\mu \mathrm{g} / \mathrm{mL})$ & $6.05 \pm 2.48$ & $6.49 \pm 2.28$ & 0.41 & $5.93 \pm 2.4$ & $5.77 \pm 1.62$ & 0.762 \\
\hline \multicolumn{7}{|l|}{ Glucose metabolism } \\
\hline Hemoglobin A1c (\%) & $5.8 \pm 0.42$ & $5.63 \pm 0.43$ & 0.074 & $6.49 \pm 0.53$ & $6.46 \pm 0.48$ & 0.854 \\
\hline Fasting glucose (mmol/L) & $5.68 \pm 0.68$ & $5.87 \pm 0.44$ & 0.145 & $6.62 \pm 0.82$ & $6.48 \pm 0.66$ & 0.418 \\
\hline 2-h glucose (mmol/L) & $7.66 \pm 2.03$ & $7.05 \pm 1.8$ & 0.144 & $12.08 \pm 3.03$ & $11.74 \pm 3.34$ & 0.661 \\
\hline Fasting insulin $(\mu \mathrm{U} / \mathrm{mL})$ & $11.58 \pm 5.79$ & $11.22 \pm 5.6$ & 0.773 & $11.49 \pm 7.12$ & $13.21 \pm 6.6$ & 0.314 \\
\hline 2-h insulin ( $\mu \mathrm{U} / \mathrm{mL})$ & $81.45 \pm 53.51$ & $82.12 \pm 71.98$ & 0.961 & $102.56 \pm 68.11$ & $109.42 \pm 71.86$ & 0.686 \\
\hline Fasting C-peptide $(\mathrm{ng} / \mathrm{mL})$ & $2.27 \pm 0.92$ & $2.29 \pm 0.81$ & 0.943 & $2.58 \pm 1.1$ & $2.81 \pm 1.26$ & 0.424 \\
\hline 2-h C-peptide (ng/mL) & $10.9 \pm 3.68$ & $10.56 \pm 3.7$ & 0.674 & $12.14 \pm 3.86$ & $12.45 \pm 4.25$ & 0.754 \\
\hline AUC glucose & $23.7 \pm 4.04$ & $23.96 \pm 3.76$ & 0.785 & $33.66 \pm 6.01$ & $32.56 \pm 5.55$ & 0.465 \\
\hline AUC insulin & $195.77 \pm 95.64$ & $208.35 \pm 105.93$ & 0.608 & $211.23 \pm 126.83$ & $260.78 \pm 129.8$ & 0.142 \\
\hline AUC C-peptide & $25.72 \pm 7.44$ & $25.47 \pm 6.22$ & 0.875 & $26.64 \pm 7.35$ & $29.86 \pm 8.47$ & 0.121 \\
\hline HOMA-IR & $2.94 \pm 1.6$ & $2.91 \pm 1.39$ & 0.937 & $3.41 \pm 2.39$ & $3.91 \pm 2.18$ & 0.383 \\
\hline HOMA- $\beta$ & $112.65 \pm 68.82$ & $104.3 \pm 97.25$ & 0.664 & $77.31 \pm 41.61$ & $87.36 \pm 38.99$ & 0.316 \\
\hline \multicolumn{7}{|l|}{ Lipids } \\
\hline Total cholesterol (mmol/L) & $5.99 \pm 1.2$ & $5.99 \pm 1.03$ & 0.996 & $6.24 \pm 1.17$ & $6.06 \pm 1.46$ & 0.57 \\
\hline Triglycerides (mmol/L) & $1.7 \pm 0.9$ & $1.69 \pm 1$ & 0.949 & $1.73 \pm 1.14$ & $1.8 \pm 1.58$ & 0.822 \\
\hline HDL cholesterol (mmol/L) & $1.45 \pm 0.39$ & $1.48 \pm 0.34$ & 0.693 & $1.48 \pm 0.37$ & $1.43 \pm 0.34$ & 0.528 \\
\hline LDL cholesterol (mmol/L) & $3.35 \pm 0.88$ & $3.28 \pm 0.82$ & 0.67 & $3.43 \pm 0.94$ & $3.33 \pm 0.99$ & 0.654 \\
\hline Apo A-1 (g/L) & $1.55 \pm 0.3$ & $1.66 \pm 0.28$ & 0.073 & $1.64 \pm 0.34$ & $1.63 \pm 0.34$ & 0.907 \\
\hline Apo B (g/L) & $1.17 \pm 0.25$ & $1.13 \pm 0.22$ & 0.506 & $1.16 \pm 0.22$ & $1.14 \pm 0.28$ & 0.836 \\
\hline LDLHDL ratio & $2.44 \pm 0.76$ & $2.34 \pm 0.82$ & 0.554 & $2.46 \pm 0.86$ & $2.46 \pm 0.94$ & 0.998 \\
\hline TGHDL ratio & $1.33 \pm 0.93$ & $1.29 \pm 1.08$ & 0.848 & $1.32 \pm 1.21$ & $1.42 \pm 1.49$ & 0.77 \\
\hline
\end{tabular}

AUC, area under the curve by 3-h oral glucose tolerance test, were calculated according the trapezoidal rule

HOMA-IR, homoeostasis model assessment of insulin resistance; HOMA-IR $=\mathrm{FIns}(\mathrm{mU} / \mathrm{mL}) \times \mathrm{FG}(\mathrm{mmol} / \mathrm{L}) / 22.5$

HOMA- $\beta$, homoeostasis model assessment of $\beta$-cell function; HOMA- $\beta=$ FIns $\times 20 /(\mathrm{FG}-3.5)$

$H D L$ high-density lipoprotein, $L D L$ low-density lipoprotein, apo $A-1$ apolipoprotein $A-1$, apo $B$ apolipoprotein $B$, TG Triglycerides

$P$ value was for the differences between the two groups, compared by independent student's $t$-test
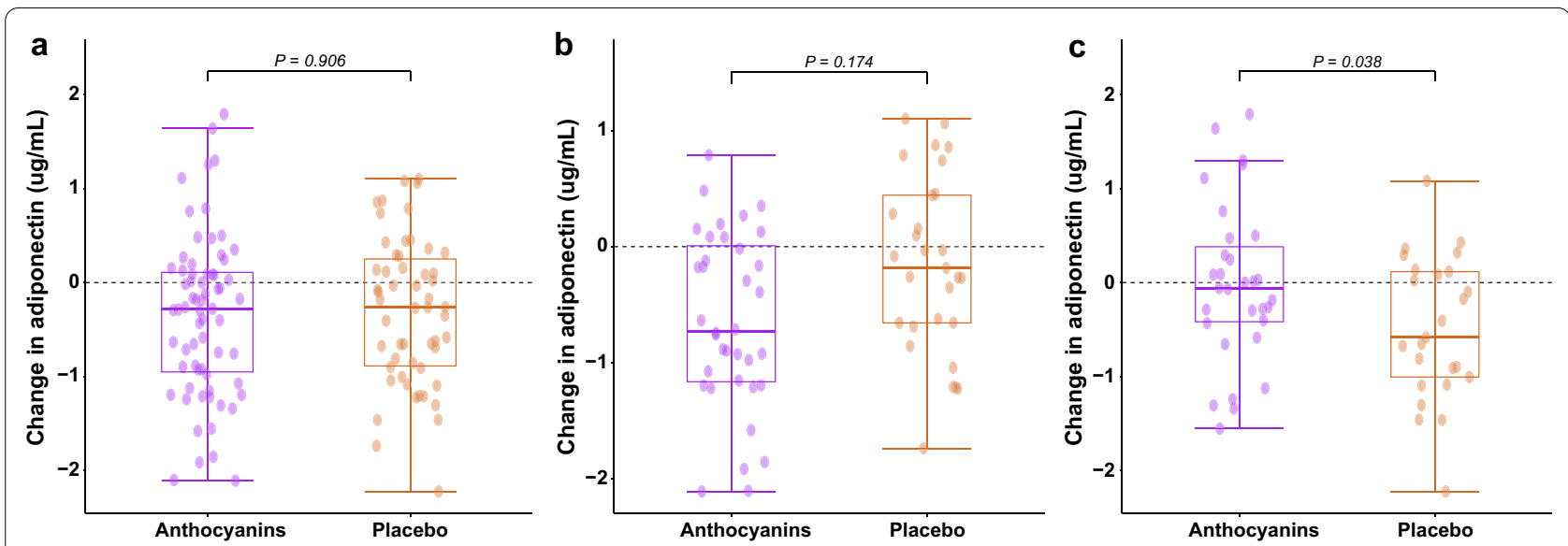

Fig. 2 Changes in serum adiponectin after 12 weeks of intervention with anthocyanins or placebo. a changes in serum adiponectin among all subjects, $\mathbf{b}$ changes in serum adiponectin in the subjects with prediabetes, $\mathbf{c}$ changes in serum adiponectin in the subjects with newly diagnosed diabetes 


\section{Discussion}

To our knowledge, this is the first study to examine the effect of supplementation with purified anthocyanins on serum adiponectin in patients with prediabetes and newly diagnosed diabetes. This study showed that supplementation with $320 \mathrm{mg} /$ day of purified anthocyanins for 12 weeks improved serum adiponectin and decreased fasting glucose, independent of hypoglycemic agents, in patients with newly diagnosed diabetes but not in patients with prediabetes.

\section{Comparison with other studies}

Few intervention studies have investigated the effect of anthocyanins on circulating adiponectin in cardiometabolic diseases. A previous trial from our laboratory conducted in patients with well-controlled type 2 diabetes showed that purified anthocyanins increased serum adiponectin and decreased fasting glucose, but potential interactions with hypoglycemic medications were not avoided [11]. Thus, we rigorously tried to ensure that our participants did not take any hypoglycemic drug or present with any other chronic inflammatory disease that could interfere with the results. In the present study, we observed significant improvements in adiponectin and fasting glucose in the participants with newly diagnosed diabetes but not in those with prediabetes, which may be due to the lower hyperglycemic status of prediabetes.

Three trials reported changes in adiponectin using anthocyanin-rich supplements in patients with metabolic syndrome. Black raspberry treatment for 12 weeks significantly improved serum adiponectin levels (net change $1.9 \mu \mathrm{g} / \mathrm{mL}$ ) and vascular endothelial function [10]. Consuming cranberry juice for 60 days increased serum adiponectin levels (graphic display) but had no effect on inflammatory markers [9]. Anthocyanin-rich grape powder increased plasma adiponectin in subjects with nondyslipidemia (net change $1.1 \mu \mathrm{g} / \mathrm{mL}$ ) but decreased plasma adiponectin in subjects with dyslipidemia (net change $-1.7 \mu \mathrm{g} / \mathrm{mL}$ ), who might have increased levels of inflammation and be less responsive to the dietary intervention [14]. Our participants had a mean triglyceride level of $1.8 \mathrm{mmol} / \mathrm{L}$ and a mean HDL level of $1.46 \mathrm{mmol} / \mathrm{L}$, with approximately $50 \%$ being within the metabolically normal range; moreover, they had a normal mean CRP level of $2.2 \mathrm{mg} / \mathrm{L}$. It is not surprising to observe a less remarkable improvement in adiponectin (net change $0.46 \mu \mathrm{g} / \mathrm{mL}$ ) in the subjects with newly diagnosed diabetes and a nonsignificant change in prediabetes and among all subjects.

\section{Possible mechanism}

It has been demonstrated that adiponectin could increase hepatic insulin sensitivity and glucose utilization through the activation of adenosine monophosphate (AMP) kinase and peroxisome proliferator-activated receptor-alpha (PPAR $\alpha)$ [15]. A previous experimental study from our laboratory showed that anthocyanin upregulated adiponectin secretion in adipocytes through the transcription factor forkhead box O1 (FoxO1) in diabetic mice [16]. Basic studies have also demonstrated the antidiabetic effect of anthocyanins by activating AMP-activated protein kinase and PPAR in adipose tissue and skeletal muscles [17]. Anthocyanin might also ameliorate insulin resistance via the activation of insulin signaling and enhanced glucose transporter 4 (GLUT4) translocation, which increases glucose uptake and reduces the hyperglycemia associated with metabolic disorders [18]. These studies may help to explain the alterations of adiponectin and fasting glucose in patients with newly diagnosed diabetes.

The potential cardiovascular-protective effect of anthocyanins is clearly understood. The study of our laboratory also found that anthocyanin protected against diabetes-related endothelial dysfunction [16]. On the other hand, hypoadiponectinemia-induced inflammasome activation may be the molecular mechanism for diabetic vascular endothelial dysfunction [19]. Basic and clinical studies have identified the anti-atherogenic function of adiponectin [20] and its positive effect on vascular inflammation and endothelial function [21]. Adiponectin could also improve endothelium-independent vasodilation by inducing the production of endothelial nitric oxide (NO) [22].

\section{Clinical implications of the findings}

In the patients with newly diagnosed diabetes, we observed a decrease in serum adiponectin in the placebo group, which revealed a change in adiponectin in the progression of T2DM, whereas anthocyanins prevented the decline in adiponectin, leading to a mild but significant relative incremental improvement. Epidemiological studies have suggested that hypoadiponectinemia is an important component of the pathogenesis in insulin resistance and T2DM [23]. A cross-sectional study suggested that decreased serum adiponectin might be an independent predictor for the progression of T2DM [24]. Some prospective studies have shown that low levels of adiponectin predict the development of insulin resistance $[25,26]$. Adiponectin has been proposed to be a novel target for the prevention and treatment of T2DM $[27,28]$. Taken together, anthocyanins may prevent the 
progression of T2DM by improving serum adiponectin and fasting glucose in newly diagnosed diabetes.

Several studies suggested that hypoadiponectinemia was associated with vascular damage and contributed to cardiovascular risk in T2DM $[29,30]$. A cohort study showed that a low level of adiponectin might predict the impairment of endothelium-independent vasodilation in newly diagnosed T2DM patients [31]. Thus, the favorable improvement in adiponectin in this study may also have a cardiovascular-protective effect, which is consistent with the clinical implications of the improvements in apo A-1 and apo B [32, 33].

\section{Limitations}

The following limitations should be considered in the present study: first, there was possible incomplete blinding in this study because of the different contents of capsules, which may have resulted in the relatively high dropout in the placebo group; second, there was no objective measure of compliance during the intervention period, and data on serum anthocyanins or their metabolites were lacking.The time required for complete clearance of anthocyanins from the circulation is $6-8 \mathrm{~h}$, which is exceeded by the $8-10 \mathrm{~h}$ of fasting [34]. Finally, we only measured total adiponectin rather than high-molecularweight adiponectin. Nonetheless, data on the relevance of their distinction showed similar results $[35,36]$.

In conclusion, this randomized placebo-controlled trial showed that 12 weeks of daily supplementation with $320 \mathrm{mg}$ anthocyanins increased serum adiponectin and decreased fasting glucose in patients with newly diagnosed diabetes but not in those with prediabetes. Additional long-term studies are needed to confirm the serial changes in adiponectin by anthocyanins intervention from normoglycemic individuals to individuals with prediabetes and new-onset diabetes.

\section{Supplementary information}

Supplementary information accompanies this paper at https://doi. org/10.1186/s12986-020-00498-0.

Additional file 1: Table S1: Baseline characteristic of the participants; Table S2: The adverse events reported in the trial; Table S3: Average daily intake of food groups and nutrients by the participants at baseline and 12 weeks; Table S4: Changes in adiponectin and glucolipid metabolic markers after 12-week intervention in subjects with newly diagnosed diabetes; Table S5: Changes in adiponectin and glucolipid metabolic markers after 12-week intervention in subjects with prediabetes.

\section{Abbreviations}

T2DM: Type 2 diabetes; RCT: Randomized controlled trial; IFG: Impaired fasting glucose; IGT: Impaired glucose tolerance; HbA1c: Glycated hemoglobin A1c; LDL: Low-density lipoprotein ; HDL: High-density lipoprotein; CRP: C-reactive protein; apoA-1: Apolipoprotein A-1; apo B: Apolipoprotein B.
Acknowledgements

The authors appreciate all the patients for their continuing participation in this study and all the medical staff for blood sample and data collection.

\section{Authors' contributions}

LY: writing the manuscript; WL: study supervision; YQ and JY: data collection and curation; LW: statistical analysis and visualization; YL: study resources; CW and JM: project administration and funding acquisition. All authors read and approved the final manuscript.

\section{Funding}

This study was supported by the Key Project of National Natural Science Foundation of China (Grant Number 81730090) and the Health Technology Project of Nanshan, Shenzhen (Grant Number 2019034).

\section{Availability of data and materials}

The datasets used and/or analyzed during the current study are available from the corresponding author on reasonable request.

Ethics approval and consent to participate

The study was approved by the Ethics Committee of Sun Yat-sen University (No. 201503), and each participant provided written informed consent.

\section{Consent for publication}

Not applicable.

\section{Competing interests}

The authors declare that they have no competing interests.

\section{Author details}

${ }^{1}$ Center for Chronic Disease Control, NanShan, Shenzhen, People's Republic of China. ${ }^{2}$ Guangdong Provincial Key Laboratory of Food, Nutrition and Health, Department of Nutrition, School of Public Health, Sun Yat-Sen University, Guangzhou, People's Republic of China.

Received: 11 May 2020 Accepted: 7 September 2020

Published online: 21 September 2020

\section{References}

1. Li S, Shin HJ, Ding EL, van Dam RM. Adiponectin levels and risk of type 2 diabetes: a systematic review and meta-analysis. JAMA. 2009;302:179-88.

2. Liu C, Feng X, Li Q, Wang Y, Li Q, Hua M. Adiponectin, TNF-alpha and inflammatory cytokines and risk of type 2 diabetes: a systematic review and meta-analysis. Cytokine. 2016;86:100-9.

3. Lopez-Jaramillo P. The role of adiponectin in cardiometabolic diseases: effects of nutritional interventions. J Nutr. 2016;146:422S-426S.

4. Katsiki N, Mantzoros C, Mikhailidis DP. Adiponectin, lipids and atherosclerosis. Curr Opin Lipidol. 2017:28:347-54.

5. Menzaghi C, Trischitta V. The adiponectin paradox for all-cause and cardiovascular mortality. Diabetes. 2018:67:12-22.

6. Schulze MB, Shai I, Rimm EB, Li T, Rifai N, Hu FB. Adiponectin and future coronary heart disease events among men with type 2 diabetes. Diabetes. 2005;54:534-9.

7. Bergmark BA, Cannon CP, White WB, Jarolim P, Liu Y, Bonaca MP, Zannad F, Morrow DA. Baseline adiponectin concentration and clinical outcomes among patients with diabetes and recent acute coronary syndrome in the EXAMINE trial. Diabetes Obes Metab. 2017;19:962-9.

8. Jennings A, Welch AA, Spector T, Macgregor A, Cassidy A. Intakes of anthocyanins and flavones are associated with biomarkers of insulin resistance and inflammation in women. J Nutr. 2014;144:202-8.

9. Simao TN, Lozovoy MA, Simao AN, Oliveira SR, Venturini D, Morimoto HK, Miglioranza LH, Dichi I. Reduced-energy cranberry juice increases folic acid and adiponectin and reduces homocysteine and oxidative stress in patients with the metabolic syndrome. Br J Nutr. 2013;1 10:1885-94.

10. Jeong HS, Hong SJ, Lee TB, Kwon JW, Jeong JT, Joo HJ, Park JH, Ahn CM, Yu CW, Lim DS. Effects of black raspberry on lipid profiles and vascular endothelial function in patients with metabolic syndrome. Phytother Res. 2014:28:1492-8. 
11. Li D, Zhang Y, Liu Y, Sun R, Xia M. Purified anthocyanin supplementation reduces dyslipidemia, enhances antioxidant capacity, and prevents insulin resistance in diabetic patients. J Nutr. 2015;145:742-8.

12. American Diabetes A. Standards of medical care in diabetes-2013. Diabetes Care. 2013;36(Suppl 1):S11-66.

13. Qin Y, Xia M, Ma J, Hao Y, Liu J, Mou H, Cao L, Ling W. Anthocyanin supplementation improves serum LDL- and $\mathrm{HDL}$-cholesterol concentrations associated with the inhibition of cholesteryl ester transfer protein in dyslipidemic subjects. Am J Clin Nutr. 2009;90:485-92.

14. Barona J, Blesso CN, Andersen CJ, Park Y, Lee J, Fernandez ML. Grape consumption increases anti-inflammatory markers and upregulates peripheral nitric oxide synthase in the absence of dyslipidemias in men with metabolic syndrome. Nutrients. 2012;4:1945-57.

15. Yamauchi T, Kamon J, Ito Y, Tsuchida A, Yokomizo T, Kita S, Sugiyama T, Miyagishi M, Hara K, Tsunoda M, et al. Cloning of adiponectin receptors that mediate antidiabetic metabolic effects. Nature. 2003;423:762-9.

16. Liu Y, Li D, Zhang Y, Sun R, Xia M. Anthocyanin increases adiponectin secretion and protects against diabetes-related endothelial dysfunction. Am J Physiol Endocrinol Metab. 2014;306:E975-988.

17. Rozanska D, Regulska-llow B. The significance of anthocyanins in the prevention and treatment of type 2 diabetes. Adv Clin Exp Med. 2018;27:135-42.

18. Luna-Vital D, Weiss M, Gonzalez de Mejia E. Anthocyanins from purple corn ameliorated tumor necrosis factor-alpha-induced inflammation and insulin resistance in 3T3-L1 adipocytes via activation of insulin signaling and enhanced GLUT4 translocation. Mol Nutr Food Res. 2017;61:1700362.

19. Zhang J, Xia L, Zhang F, Zhu D, Xin C, Wang H, Zhang F, Guo X, Lee Y, Zhang $L$, et al. A novel mechanism of diabetic vascular endothelial dysfunction: hypoadiponectinemia-induced NLRP3 inflammasome activation. Biochem Biophys Acta. 2017;1863:1556-67.

20. Kishida K, Funahashi T, Shimomura I. Molecular mechanisms of diabetes and atherosclerosis: role of adiponectin. Endocr Metab Immune Disord Drug Targets. 2012;12:118-31.

21. Han SH, Sakuma I, Shin EK, Koh KK. Antiatherosclerotic and anti-insulin resistance effects of adiponectin: basic and clinical studies. Prog Cardiovasc Dis. 2009;52:126-40.

22. Xi W, Satoh H, Kase H, Suzuki K, Hattori Y. Stimulated HSP90 binding to eNOS and activation of the PI3-Akt pathway contribute to globular adiponectin-induced NO production: vasorelaxation in response to globular adiponectin. Biochem Biophys Res Commun. 2005;332:200-5.

23. Kadowaki T, Yamauchi T, Kubota N, Hara K, Ueki K, Tobe K. Adiponectin and adiponectin receptors in insulin resistance, diabetes, and the metabolic syndrome. J Clin Invest. 2006;116:1784-92.

24. Banerjee A, Khemka VK, Roy D, Poddar J, Roy TKS, Karnam SA. Role of serum adiponectin and vitamin D in prediabetes and diabetes mellitus. Can J Diab. 2017:41:259-65.

25. Lindsay RS, Funahashi T, Hanson RL, Matsuzawa Y, Tanaka S, Tataranni PA, Knowler WC, Krakoff J. Adiponectin and development of type 2 diabetes in the Pima Indian population. Lancet. 2002;360:57-8.
26. Jalovaara K, Santaniemi M, Timonen M, Jokelainen J, Kesaniemi YA, Ukkola O, Keinanen-Kiukaanniemi S, Rajala U. Low serum adiponectin level as a predictor of impaired glucose regulation and type 2 diabetes mellitus in a middle-aged Finnish population. Metabolism. 2008;57:1130-4.

27. Achari $A E$, Jain SK. Adiponectin, a therapeutic target for obesity, diabetes, and endothelial dysfunction. Int J Mol Sci. 2017;18(6):1321.

28. Hafiane A, Gasbarrino K, Daskalopoulou SS. The role of adiponectin in cholesterol efflux and HDL biogenesis and metabolism. Metabolism. 2019;100:153953.

29. Spurna J, Karasek D, Kubickova V, Goldmannova D, Krystynik O, Schovanek J, Zadrazil J. Relationship of selected adipokines with markers of vascular damage in patients with type 2 diabetes. Metab Syndr Relat Disord. 2018;16:246-53.

30. Stojanovic S, Ilic MD, Ilic S, Petrovic D, Djukic S. The significance of adiponectin as a biomarker in metabolic syndrome and/or coronary artery disease. Vojnosanit Pregl. 2015;72:779-84.

31. Li H, Xiao Y, Liu H, Chen XY, Li XY, Tang WL, Liu SP, Xu AM, Zhou ZG. Hypoadiponectinemia predicts impaired endothelium-independent vasodilation in newly diagnosed type 2 diabetic patients: an 8-year prospective study. Chin Med J (Engl). 2011;124:3607-12.

32. Walldius $G$, Jungner I, Aastveit AH, Holme I, Furberg CD, Sniderman AD. The apoB/apoA-I ratio is better than the cholesterol ratios to estimate the balance between plasma proatherogenic and antiatherogenic lipoproteins and to predict coronary risk. Clin Chem Lab Med. 2004;42:1355-63.

33. McQueen MJ, Hawken S, Wang X, Ounpuu S, Sniderman A, Probstfield J, Steyn K, Sanderson JE, Hasani M, Volkova E, et al. Lipids, lipoproteins, and apolipoproteins as risk markers of myocardial infarction in 52 countries (the INTERHEART study): a case-control study. Lancet (London, England). 2008;372:224-33.

34. McGhie TK, Walton MC. The bioavailability and absorption of anthocyanins: towards a better understanding. Mol Nutr Food Res. 2007:51:702-13.

35. Kizer JR, Benkeser D, Arnold AM, Mukamal KJ, Ix JH, Zieman SJ, Siscovick DS, Tracy RP, Mantzoros CS, Defilippi CR, et al. Associations of total and high-molecular-weight adiponectin with all-cause and cardiovascular mortality in older persons: the Cardiovascular Health Study. Circulation. 2012;126:2951-61.

36. Kizer JR, Arnold AM, Benkeser D, Ix JH, Djousse L, Zieman SJ, Barzilay Jl, Tracy RP, Mantzoros CS, Siscovick DS, Mukamal KJ. Total and high-molecular-weight adiponectin and risk of incident diabetes in older people. Diabetes Care. 2012;35:415-23.

\section{Publisher's Note}

Springer Nature remains neutral with regard to jurisdictional claims in published maps and institutional affiliations.

Ready to submit your research? Choose BMC and benefit from

- fast, convenient online submission

- thorough peer review by experienced researchers in your field

- rapid publication on acceptance

- support for research data, including large and complex data types

- gold Open Access which fosters wider collaboration and increased citations

- maximum visibility for your research: over $100 \mathrm{M}$ website views per year

At BMC, research is always in progress.

Learn more biomedcentral.com/submissions 